\title{
Calidad como demanda intangible de mercado y organizaciones sustentables
}

\section{Quality as an intangible market demand and sustainable organizations}

\author{
Mayra Lucía Maycotte de la Peña ${ }^{1}$ \\ Centro de Investigación en Alimentación y Desarrollo \\ Imaycotte.419@estudiantes.ciad.mx \\ Jesús Martín Robles Parra ${ }^{2 *}$ \\ Centro de Investigación en Alimentación y Desarrollo \\ jrobles@ciad.mx \\ Martín Ernesto Tiznado Hernández ${ }^{3}$ \\ Centro de Investigación en Alimentación y Desarrollo \\ tiznado@ciad.mx

\section{Martín Preciado Rodríguez ${ }^{2}$ \\ Centro de Investigación en Alimentación y Desarrollo mpreciado@ciad.mx} \\ Julio César Tafolla Arellano ${ }^{4}$ \\ Universidad Autónoma Agraria Antonio Narro \\ jtafare@uaaan.edu.mx

\section{Karen Montaño Silva ${ }^{5}$} \\ Universidad Estatal de Sonora \\ karen.montano@ues.mx
}

* Autor corresponsal.

1 Centro de Investigación en Alimentación y Desarrollo. Coordinación de Desarrollo Regional. Carretera Custavo Enrique Astiazarán Rosas 46. Hermosillo. Sonora. MÉXICO.

2 Centro de Investigación en Alimentación y Desarrollo. Departamento de Economía de la Coordinación de Desarrollo Regional. Carretera Gustavo Enrique Astiazarán Rosas 46. Hermosillo, Sonora, MÉXICO.

3 Centro de Investigación en Alimentación y Desarrollo. Departamento de Biotecnología de la Coordinación de Tecnología de Alimentos de Origen Vegetal. Carretero Gustavo Enrique Astiazarán Rosas 46, Hermosillo, Sonora, MEXICO.

4 Universidad Autónoma Agraria Antonio Narro. Departamento de Ciencias Básicas. Calzada Antonio Narro 1923. Saltillo, Coahuila, MÉXICO.

5 Universidad Estatal de Sonora. Departamento de Gestión y Desarrollo de Negocios. Blvd. Rosales 189, Hermosillo, Sonora, MÉXICO

\section{Resumen}

Propósito: Recopilar, analizar y sistematizar la información publicada sobre el estado del conocimiento de temas de calidad, demanda intangible de mercado y organizaciones sustentables, así como discutir, a partir de los resultados, la asociación entre calidad y demanda intangible de mercado y su relación con las organizaciones sustentables.

Diseño/Metodología: Es un estudio exploratorio. Parte de una profunda revisión de literatura en diversas bases de datos, selección, clasificación de documentos y lectura crítica de textos.

Resultados: El análisis y sistematización obtenidos indican una asociación entre los conceptos y se configuran los constructos teóricos calidad, calidad como demanda intangible de mercado y organización sustentable, basados en la sistematización de la información obtenida de la selección de documentos que conforman este estudio.

Implicaciones prácticas: Los resultados aportan evidencia de la relación entre calidad y sustentabilidad que proporciona conocimientos para abordar problemáticas de las organizaciones modernas, de ahí la importancia de esta línea de investigación.

Limitaciones: Debido a la naturaleza exploratoria del artículo, la principal limitación son las dimensiones de las bases de datos consultadas. Con respecto a los límites de este estudio están demarcados alcances analíticos del concepto compuesto calidad e intangibilidad, demanda de mercado y organización sustentable.

Originalidad/Valor: Esta investigación permite comprender y aportar elementos conceptuales al estudio de las organizaciones en su tránsito hacia organizaciones sustentables, sobre la base de las demandas intangibles de mercado.
INFORMACIÓN ARTÍCULO

Recibido: 28 de Octubre 2021

Aceptado: 21 de Diciembre 2021
Palabras Claves:

Demanda intangible de mercado

Consumo responsable

Organización sustentable

Responsabilidad social 


\begin{abstract}
Purpose: Collect, analyze, and systematize the information published on the state of the art of quality issues, intangible market demand and sustainable organizations, as well as discuss the association between quality and intangible market demand and its relationship with the sustainable organizations.

Design/Methodology: It is an exploratory study. Part of a deep literature review in various databases, selection, classification of documents and critical reading of texts.

Findings: The analysis and systematization obtained indicate an association between the concepts and the theoretical constructs of quality, quality as intangible market demand and sustainable organization are configured based on the systematization of the information obtained from the selection of documents that make up this study.

Practical implications: The results provide evidence of the relationship between quality and sustainability that provides knowledge to address problems in modern organizations, hence the importance of this line of investigation.

Limitations: Due to the exploratory nature of the article, the main limitation is the dimensions of the databases consulted. Regarding the limits of this study, analytical scopes of the composite concept of quality and intangibility, market demand and sustainable organization are demarcated. Originality/Value: This research allows us to understand and contribute conceptual elements to the study of organizations in their transition to sustainable organizations, based on intangible market demands.
\end{abstract}

\section{ARTICLE INFO}

Received: 28 October 2021

Accepted: 21 December 2021
Keywords:

Intangible market demand Responsible consumption Sustainable organization Social responsibility

\section{INTRODUCCIÓN}

Actualmente, las organizaciones se encuentran inmersas en un ambiente dinámico y de constantes cambios, en donde la calidad y la adopción de prácticas de sustentabilidad económica, social y ambiental juegan un papel importante como ventajas competitivas para permanecer en el mercado. Las distintas concepciones de calidad evidencian que esta no es un concepto estático, pues ha cambiado a lo largo del tiempo. No existe una definición única en la literatura porque depende del momento de la historia que se ha vivido y de las necesidades de los distintos grupos de interés que están alrededor de una organización, entre ellos los consumidores, empleados, directivos, proveedores, entre otros. El concepto de calidad en las organizaciones ha evolucionado. Algunas definiciones que han dado los pioneros en esta área son: "adecuación al uso y ausencia de defectos" (Juran, 1990), "satisfacción de las necesidades del consumidor" (Deming, 1989), "cumplimiento de las especificaciones" (Crosby, 1987), "la mínima pérdida que el uso de un producto o servicio causa a la sociedad" (Taguchi, 1986); definiciones citadas por Cuatrecasas y González (2017).

La Organización Internacional de Estandarización (International Organization for Standarization 112
ISO, s.f.) menciona que "la calidad es el grado en el que un conjunto de características inherentes cumple con los requisitos" ( $p$ ).

Por su parte, Cantú (2011) define calidad como "todas las cualidades con que cuenta un producto -o un servicio- para ser de utilidad a quien lo emplea" (p.3), muy en sintonía a Cuatrecasas y González (2017) quienes afirman que la calidad puede definirse como el conjunto de características que posee un producto o servicio, así como su capacidad de satisfacción de los requerimientos del usuario.

Estas concepciones de calidad destacan la satisfacción de las exigencias del cliente sin especificar si es por los atributos tangibles o intangibles del producto. Las demandas que hacen los consumidores cada vez más preparados e informados se han convertido en exigencias complejas que no tienen que ver solamente con atributos tangibles del producto, sino con características no visibles del mismo que están relacionadas con la sustentabilidad económica, social, ambiental o con demandas de responsabilidad social. La complejidad del entorno, la globalización y los cambios sociales están afectando a las organizaciones, 
las cuales deben estar constantemente en busca de estrategias que permitan la satisfacción de las demandas de calidad y sustentabilidad de los consumidores (Hernández y Villaseñor, 2014; Solís, Robles, Preciado y Hurtado, 2017).

Este artículo, tiene el propósito de recopilar, analizar y sistematizar la información publicada en la última década sobre el estado del conocimiento en temas de calidad, demanda intangible de mercado y organizaciones sustentables, así como discutir la asociación entre estos constructos teóricos. Es interesante analizar si la calidad puede ser configurada como una demanda intangible de mercado, es decir, cómo se han incorporado los aspectos económico, social y ambiental al concepto de calidad, y, a su vez, si este concepto integrado -calidad como demanda intangible de mercado- tiene relación con los cambios de paradigma en las organizaciones modernas que las llevan a establecer prácticas de sustentabilidad. En otras palabras, lo que las lleva a configurarse como organizaciones sustentables.

En la segunda sección de este documento se expone la metodología utilizada, basada en un estudio exploratorio de recopilación, análisis, sistematización y lectura crítica de textos. En la tercera sección se expone la revisión de literatura acerca de los conceptos teóricos: calidad, demanda intangible de mercado y organización sustentable. En la cuarta sección se discuten los resultados de la revisión de literatura, y, en la quinta sección se enuncian los comentarios finales.

\section{METODOLOGÍA}

Esta investigación se basa en un trabajo exploratorio mediante el uso de técnicas de selección, clasificación y lectura crítica de textos de manera sistemática y objetiva. Se divide en dos etapas: primeramente, la referente a la investigación sobre el concepto compuesto calidad como demanda intangible de mercado, que pretende evidenciar si existe literatura que aborde dicha construcción teórica. Esta exploración se realizó en cinco bases de datos, analizándose el título, resumen y palabras clave. Las rutas de búsqueda fueron tanto en español como inglés: "demanda de mercado", posteriormente "demanda intangible de mercado" y finalmente "calidad como demanda intangible de mercado". Cuando se estableció el primer descriptor, las bases de datos mostraron una amplia cantidad de artículos abordando el concepto. Cuando se estableció el segundo descriptor, la base referencial fue muy limitada. Para finalizar, se estableció el tercer descriptor, donde se vincularon los conceptos de calidad y demanda intangible de mercado, del cual se encontraron nulas referencias (ver Tabla 1).

Tabla 1. Primera ruta de búsqueda.

\begin{tabular}{lccc}
\hline & & Descriptor o palabra clave & \\
\cline { 2 - 4 } Base de datos & Demanda de mercado & $\begin{array}{c}\text { Demanda intangible de } \\
\text { mercado }\end{array}$ & $\begin{array}{c}\text { Calidad como demanda } \\
\text { intangible de mercado }\end{array}$ \\
\hline Scopus & 14834 & 0 & 0 \\
Science Direct & 42509 & 1 & 0 \\
Redalyc & 4331 & 0 & 0 \\
ScieLO & 132 & 0 & 0 \\
Google Académico & 21800 & 1 & 0 \\
\hline
\end{tabular}

Fuente: elaboración propia.

La segunda etapa de esta investigación parte del hallazgo de limitadas referencias sobre el concepto compuesto calidad como demanda intangible de mercado, por lo que la segunda ruta de búsqueda para la revisión de literatura se hace sobre los conceptos tomados de forma individual: calidad, demanda intangible de mercado y organización sustentable. Las fuentes de información consistieron en libros, artículos científicos, actas de simposios y conferencias, tesis y sitios web de instituciones reconocidas, obteniendo con ello una base de datos de 233 documentos. Posteriormente, se acotó la búsqueda descartando los documentos que no cumplieran con los criterios de inclusión que se muestran en la Tabla 2. 
Tabla 2. Criterios de inclusión.

\begin{tabular}{ll}
\hline Concepto teórico & \multicolumn{1}{c}{ Criterios de inclusión } \\
\hline Calidad & $\begin{array}{l}\text { Atributos tangibles e intangibles, decisiones de compra, calidad en las organizaciones, cali- } \\
\text { dad y sustentabilidad, calidad total, certificaciones de calidad, cultura de calidad, disposición } \\
\text { a pagar por la calidad, evolución de la calidad, gestión de la calidad, percepción de la calidad, } \\
\text { pioneros de la calidad, satisfacción del consumidor. }\end{array}$ \\
$\begin{array}{l}\text { Demanda intangible de } \\
\text { mercado }\end{array}$ & $\begin{array}{l}\text { Consumidores, consumo sustentable, consumo socialmente responsable, comportamiento } \\
\text { ético, decisiones de compra, demandas de sustentabilidad, grupos de interés, mercado, prácti- } \\
\text { cas económicas/sociales/ambientales, responsabilidad social y marketing. }\end{array}$ \\
Organización sustentable & $\begin{array}{l}\text { Cambio de paradigma, cultura organizacional, desarrollo sustentable, diseño organizativo, for- } \\
\text { mas organizativas, impacto a la comunidad, organización, responsabilidad social empresarial, } \\
\text { responsabilidad social corporativa, sustentabilidad corporativa, sustentabilidad económica/ } \\
\text { social/ambiental, teorías de la organización. }\end{array}$ \\
\hline
\end{tabular}

Fuente: elaboración propia.

El resultado arrojó una base de datos de 50 documentos. Ver la segunda ruta de búsqueda en la Figura 1.

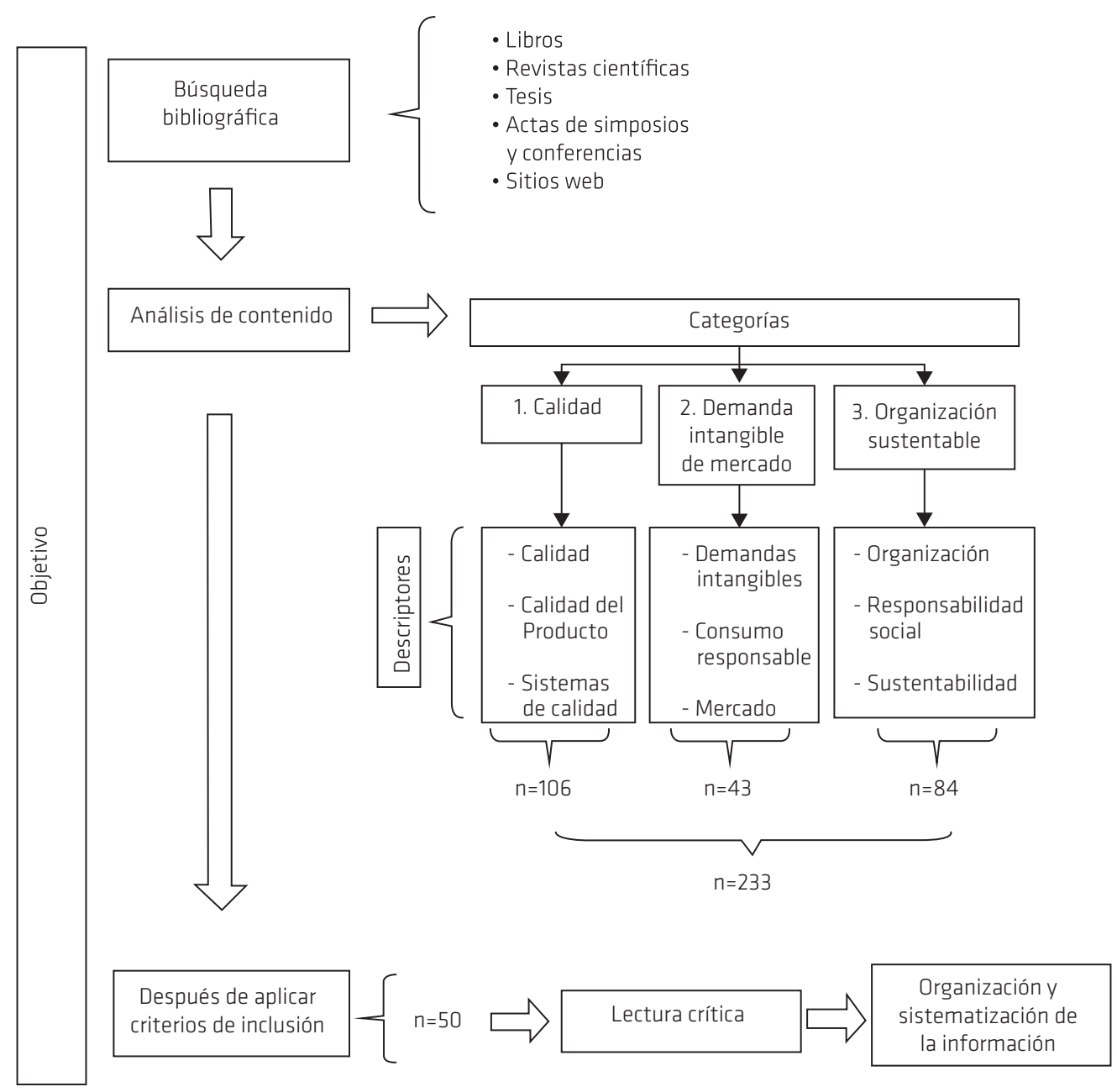

Figura 1. Segunda ruta de búsqueda. Fuente: elaboración propia. 


\section{REVISIÓN DE LITERATURA}

\section{El concepto de calidad}

El concepto de calidad es un concepto dinámico, pues no existe una definición mejor que otra porque va adecuando y variando su orientación según las exigencias del mercado en cada momento (Cuatrecasas y González, 2017).

Autores como Cantú (2011), Cuatrecasas y González (2017), Gutiérrez (2014) y Platas y Cervantes (2017) hacen una descripción de las principales etapas en la evolución del concepto de calidad: la primera etapa fue la de inspección, esta se dio una vez establecida la producción en masa después de la Revolución Industrial, la cual se distinguía por actividades como la detección y solución de problemas a través de la medición y las pruebas a los productos para evitar que llegaran a manos del consumidor con defectos. Los inspectores eran los encargados de medir y contrastar las características del producto con las especificadas por el cliente, desechando o reprocesando el producto defectuoso.

Posteriormente, se dio la etapa de control de calidad hacia la década de 1930, etapa en que la inspección dejó de ser masiva para pasar a ser por muestreos. Se utilizaron métodos de control estadístico como los gráficos de control iniciados por Shewhart, que entendía la calidad como "un proceso de variación que se podía controlar y prevenir" (Cantú, 2011, p.5), lo que originó el desarrollo de técnicas de Control Estadístico de la Calidad para grandes volúmenes de producción, con la finalidad de cumplir con las especificaciones tangibles del producto.

Para la década de 1950 la etapa del aseguramiento de calidad se tornó importante en las organizaciones (Cantú, 2011). Durante esta fase se promovió la prevención y no tanto la detección de fallas. Para un mejor control de calidad se hace necesario involucrar a todos los departamentos de la organización para el establecimiento de políticas de calidad, sobre las acciones sistemáticas planeadas para enfocarse no solo en la manufactura sino también en el servicio (Cuatrecasas y Conzález, 2017; Platas y Cervantes, 2017).

De los años sesenta y hasta 1990, las funciones de la calidad pasaron a un sistema de Control Total de la Calidad (Total Quality Control, TQC) de productos y servicios, donde se involucraba a toda la organización en la creación de calidad, no solo al área de producción. Surgen los organismos verificadores y certificadores de calidad como la ISO para generar confianza en los clientes acerca del producto y su proceso de fabricación. En 1990, debido a la globalización, surge la Administración por Calidad Total (Total Quality Management, TQM) que plantea un cambio de filosofía, de cultura y de liderazgo en la organización, enfocándose en las necesidades del cliente y observando las tendencias del mercado, involucrando a la alta dirección para convertir la calidad en una estrategia para la competitividad. Se adoptan modelos de excelencia de calidad total y se elaboran planes estratégicos para llevar a la organización a un nivel de permanencia y crecimiento. Los aportes de Ishikawa son importantes en esta etapa, porque su filosofía se basa en que la calidad es primero antes que las utilidades, la orientación al consumidor, el respeto al ser humano, el involucramiento y reconocimiento de todos los empleados, la mejora permanente, entre otras. Reconoce que las mejoras pueden provenir incluso de los empleados, pues la calidad es responsabilidad de todos y no solo de un área de la organización. En esta etapa, las organizaciones han logrado niveles similares de calidad por lo que posteriormente se buscarán nuevas ventajas competitivas (Cantú, 2011; Gutiérrez, 2014).

Cantú (2011) añade una quinta etapa, la de innovación y tecnología que inicia con el siglo XXI, en la que las organizaciones están en constante innovación (de productos, procesos y servicios) y adaptación mediante el uso de tecnologías de procesos e información para responder rápidamente a las variaciones del mercado y las tendencias económicas en el mundo, así como a los cambios políticos y sociales, buscando mantenerse competitivas mediante productos y servicios diferenciados.

En esta etapa la competencia es muy fuerte y en ocasiones devastadora, de manera que la ventaja competitiva puede no ser duradera. Una organización, en esta fase, que visualiza establecerse en una comunidad, busca la forma de ganarse su confianza invirtiendo en investigación para estrechar vínculos y para el desarrollo regional, ampliando la visión de crecimiento económico a otros aspectos importantes para las personas como son lo social y lo ambiental - dimensiones referidas a la sustentabilidad- (Sanabria, Romero y Flórez, 2014). 
Existe un grupo creciente de autores que toma en cuenta los aspectos de responsabilidad social o demandas intangibles de los consumidores, relacionados a la sustentabilidad social, económica y ambiental, como parte del concepto de calidad. En esta línea, se define calidad como el conjunto de características de un producto que satisface las exigencias explícitas e implícitas del cliente, es decir, aquellas que son tangibles e intangibles (Cuatrecasas y González, 2017; Gutiérrez, 2014; Kotler y Keller, 2012; Sanabria y cols., 2014).

Para Hernández y Villaseñor (2014), es importante dirigir la mirada hacia los movimientos de los consumidores que cuestionan el uso de hormonas y químicos aplicados en plantas y animales, y a los movimientos a favor de los derechos de los trabajadores, de los animales y de los pueblos, así como a las exigencias de salud, calidad de vida y medio ambiente.

Para Schönsleben (2019), la línea entre lo tangible y lo intangible se hace cada vez más difusa. Anteriormente, se percibía un producto como algo tangible que se podía ver, tocar, oler y probar, y un servicio como algo intangible. Lo que el cliente percibe actualmente como valor depende de varias características tangibles e intangibles que juntas ofrecen al consumidor los beneficios deseados. Para los bienes en particular, la tangibilidad no es suficiente, puesto que el factor decisivo que influencia la compra de un bien es toda la experiencia holística de experiencias pasadas. La decisión de compra por parte del consumidor dependerá, entonces, de una serie de factores que tiene que ver con elementos subjetivos como los aspectos psicológicos, culturales y socio- demográficos (Kotler y Keller, 2012).

En este sentido, la Teoría de las Convenciones abona sobre el proceso de valoración de la calidad que hacen los consumidores al momento de su compra (Cidell, 2012; Rossi, Devós, De Oliveira y Baker, 2020). Ruiz, Castelló, Climent, Escalona, Hernández, Loscertales y Frutos (2013) abonan que esta teoría fue propuesta por Boltanski y Thévenot (1991) y clasifica en seis las "convenciones o justificaciones" (p. 236) que definen la calidad de un producto, dado que los individuos tienen diferentes motivaciones para calificar o juzgar la calidad, y las enlistan de la siguiente manera:

1) Convenciones inspiracionales: los objetos son juzgados para ser valiosos si son únicos y creativos.

2) Convenciones de opinión: la calidad es valorada por la fama y reconocimiento de los productos.
3) Convenciones domésticas: la calidad de los productos es valorada por la confianza que les da a los consumidores el hecho de que el producto sea elaborado en un lugar específico, utilizando materias primas locales o métodos tradicionales, como las denominaciones de origen.

4) Convenciones cívicas: la calidad de los productos es evaluada en términos de la aportación al bienestar general de la sociedad, incluyendo principios éticos, aspectos de salud, medio ambiente e información al consumidor.

5) Convenciones de mercado: la calidad de los productos puede ser evaluada por el precio según el mercado.

6) Convenciones industriales: la calidad del producto se evalúa en base a parámetros técnicos objetivos y medibles, certificaciones, normas, estándares y procedimientos.

Para Rashid y Byun, citado en Araya y Rojas (2020), esta teoría puede explicar la elaboración de productos diferenciados, nuevos, alternativos y la producción localizada y especializada que busca satisfacer a un grupo de consumidores responsables que se preocupa por el comercio justo, los derechos humanos, desarrollo armamentista, procedencia del producto y que está dispuesto a pagar más por productos que cumplan sus gustos personales y exigencias de salud (Ruiz y cols., 2013), pero también esta teoría puede explicar que las organizaciones ya existentes pueden ser transformadas por nuevas convenciones (Cidell, 2012).

En tanto, Contreras-Valenzuela, Preciado-Rodríguez, Báez-Sañudo, Robles-Parra, Taddei-Bringas y Valderrain (2018) concluyen en su estudio que las certificaciones (convenciones industriales) son adoptadas por las organizaciones como una conducta estratégica para posicionarse en el mercado al tiempo que demuestran que su producto cumple con los requisitos exigidos por el cliente. En este sentido, Araya-Pizarro y Araya (2020) dan suma importancia a las certificaciones de calidad del producto, las cuales permiten crear confianza y tranquilidad en los consumidores al momento de tomar la decisión de compra, sobre todo si se trata de productos agroalimentarios. Sin embargo, tradicionalmente la calidad ha sido expresada como un resultado tangible -un número, una forma, un reporte- por lo cual, Liyanagel y Warahena (2019) sostienen que la calidad va más allá, pues corresponde a un impacto en la comunidad. 
La dimensión ética y la contribución a la sociedad son dos factores que juegan un papel importante, porque son los intangibles que debieran ser incluidos en los procesos de aseguramiento de la calidad.

Para Giménez, Jiménez y Martínez (2014), las dimensiones intangibles son las principales responsables del éxito de la calidad. Los atributos de calidad son ahora también subjetivos, cambiantes y distintos para cada grupo de interés. En este medio complejo, la calidad ha de ser entendida como una estrategia competitiva que sirve de guía a la organización para enfocar todas sus acciones hacia la adaptación a las exigencias de sus grupos de interés, su objetivo final y la permanencia en el mercado. Así, la calidad debe estar inmersa en todos los procesos de la organización y ello contribuirá a su sostenibilidad en el tiempo (Sanabria y cols., 2014).

\section{Demanda intangible de mercado}

Para definir la demanda intangible de mercado es importante precisar primero este concepto. Definir el mercado en el que una organización compite es muy importante, porque una correcta definición de este permite saber quiénes son los consumidores, competidores y demás grupos de interés afectados por la organización. Para Tarziján y Paredes (2012), el mercado es la instancia donde se negocia solo un bien o servicio. Es cuando confluye un conjunto de productos que, como sustitutos, se afectan significativamente.

Algo similar aporta Fisher y Espejo (2011) al hablar también del mercado "desde el punto de vista de la economía, (...), es el lugar donde se reúnen oferentes y demandantes y donde se determinan los precios de los bienes y servicios a través del comportamiento de la oferta y la demanda" (p.58). En tanto, para Mankiw (2017) el mercado está formado por un grupo que determina la demanda -los compradores- y otro que determina la oferta -los vendedores- de bienes y servicios.

Las definiciones anteriores muestran similitudes en cuanto a que el mercado es un grupo de compradores y vendedores, y es donde se determina el comportamiento de oferta y demanda. Dentro de estos grupos se encuentran los consumidores, quienes exigen a las organizaciones adaptarse a sus nuevas solicitudes. En esta dirección es que Solís y cols. (2017) y Terán, Robles, Preciado y López (2019) definen como demanda intangi- ble de mercado aquella exigencia que tiene que ver con aspectos no visibles en el producto final, y, que están relacionados con la sustentabilidad económica, social y ambiental. Para Zinoubi (2020), esto significa que los consumidores se han vuelto más demandantes acerca de lo que ellos compran y consumen.

En esta línea, Contreras-Valenzuela y cols. (2018) sostienen que el mercado se ha vuelto más dinámico y los consumidores más selectivos al momento de comprar, considerando atributos del producto y el comportamiento ético de la empresa. Amezcua, Briseño, Ríos y Ayala (2018), afirman que muchas campañas de mercadotecnia están alineadas con prácticas de responsabilidad social, económica y ambiental, utilizadas como estrategias para diferenciar su marca y posicionarse en la preferencia de los consumidores. Algunas investigaciones demuestran que este tipo de campañas se ha incrementado debido a que el consumidor evalúa en conjunto los atributos al momento de la decisión de compra, haciendo un juicio de valor sobre la calidad del producto según aspectos intangibles como la marca, el precio, la reputación de la empresa, y tangibles como la forma, color, sabor, tamaño, entre otros, y puede llegar a pagar un sobreprecio por productos que perciba como socialmente responsables, sobre todo si el beneficio es para una causa social.

Fischer y Espejo (2011) y Kotler y Keller (2012), precisan que la decisión de compra de los consumidores está basada en aspectos internos como externos. Entre los aspectos internos sobresalen la personalidad, las experiencias anteriores, las emociones y la percepción acerca de los productos o servicios. Entre los segundos, destacan el nivel de ingreso, la edad, el nivel educativo, los grupos de amistades y familiares, estatus laboral, entre otros. Al igual que el consumidor se ha vuelto más informado y consciente en sus comparas, el marketing ha evolucionado para adaptarse a sus nuevas demandas, dando paso a la creación de experiencias a través del marketing sensorial, el cual posibilita un nexo emocional con el cliente a través de sus vivencias y emociones que influyen en su comportamiento de compra (Palma-Perez, Arteaga-Flores, Ponce-Andrade, Loor-Chávez, Calderón y Mejía, 2018).

Sin embargo, Araya y Rojas (2020) sostienen que a pesar de la existencia de un consumidor más consciente de sus compras y que percibe un mayor beneficio de las empresas socialmente responsables, esto no necesariamente se traduce en un consu- 
mo, porque mucho dependerá del poder adquisitivo del consumidor, sus hábitos y actitudes.

Algunas de las demandas de responsabilidad social que los consumidores hacen a las organizaciones (o demandas intangibles de mercado) encontradas en la revisión de literatura se citan a continuación. En cuanto a la dimensión económica de la sustentabilidad, esta invita a buscar un bienestar económico en los grupos de interés y en la economía de la región, desarrollo de proveedores, adhesión a normas y certificaciones (Lizcano-Prada y Lombana, 2018), prácticas empresariales de colaboración (Esparza y Reyes, 2019), innovación y tecnología (Gavito y cols., 2017), buenos salarios a la comunidad, mejora de la calidad de vida de empleados y comunidad, y mayores niveles de productividad (Zarta, 2018).

En la dimensión social: la equidad de género (Dempsey, Bramley, Power y Brown, 2011; Terán y cols., 2019), apoyo con la formación de sus empleados, incorporación de los intereses de la comunidad (Esparza y Reyes, 2019), impacto a la comunidad y condiciones favorables para los trabajadores (Pérez-Batres, Miller y Pisani, 2011; Aguilera y Puerto, 2012), comercio justo, inocuidad de los alimentos, producción responsable (Lizcano-Prada y Lombana, 2018), inclusión de personas con discapacidad (Gómez y Peñaranda, 2019), protección a los consumidores (Daft, 2015), trato digno a los trabajadores y clientes, y cohesión comunitaria (Zarta, 2018).

Acerca de la dimensión ambiental, las exigencias del mercado están en función de que se refleje la gestión como un principio interiorizado por las organizaciones con relación al uso de recursos naturales de forma eficiente, gestión de suelos y uso de agua (Lizcano-Prada y Lombana, 2018), prácticas de producción más limpia (Calderón y Flórez, 2015), cumplir y exceder las normas legales, reutilizar y reciclar materiales, uso de tecnologías alternativas, empaques reciclables (Esparza y Reyes, 2019), uso de hormonas y químicos, pesticidas en la agricultura y ganadería (Hernández y Villaseñor, 2014; Turgut, Ornek y Cutright, 2011), preservación y cuidado del medio ambiente, disminución de contaminación ambiental, uso de tecnologías limpias (Zarta, 2018), fuentes de energías de baja contaminación y energías renovables (Fouquet, 2012).

\section{Organización sustentable}

Desde la mirada de Solís y cols. (2017), una organización sustentable es aquella que ha integrado los tres principios éticos del desarrollo sustentable (económico, social y ambiental) debido a la presión de grupos de consumidores, especialmente de aquellos ubicados en ciertas áreas geográficas y que han adoptado conductas de consumo en las que demandan a las organizaciones que se ajusten a los parámetros de sustentabilidad. Postura que comparten Zenteno-Hidalgo y Durán (2016) y además sostienen que el factor social dentro de las organizaciones sustentables ha tomado relevancia recientemente.

Velázquez y Vargas-Hernández (2012), destacan la definición de desarrollo sustentable dada por la Unión Internacional para la Conservación de la Naturaleza, la cual señala que "el desarrollo sustentable es un proceso de mejoría económica y social que satisface las necesidades y valores de todos los grupos interesados, manteniendo las opciones futuras y conservando los recursos naturales (p.100). En opinión de Zarta (2018), el concepto de desarrollo sustentable o sostenible a más de 30 años de haber aparecido a raíz del Informe Brundtland por la Comisión Mundial sobre el Medio Ambiente de las Naciones Unidas, sigue en construcción porque se deben contemplar en este concepto los cambios en el desarrollo de las sociedades contemporáneas respecto a sus valores, sobre la comprensión de que los recursos naturales son limitados y el crecimiento poblacional ilimitado y que implica un desarrollo armonioso en los aspectos económico, social y ambiental.

Kent (2020), parte de la premisa de que una organización sustentable es aquella culturalmente responsable, es decir, aquella que reconoce la responsabilidad social, individual y colectiva, una vez que logre internalizar los valores éticos de una cultura sustentable. Por su parte, Robles y Garza (2011) afirman que los mercados tienden a ser cada vez más dinámicos, al tiempo que la competitividad impone un creciente número de exigencias o condicionantes a las organizaciones. Por ejemplo, en el sistema vid de mesa en el estado de Sonora, las condiciones de mercado se expresan en exigencias sobre regulaciones fitosanitarias, actividades de control de calidad y principios de Responsabilidad Social Empresarial (RSE). Las organizaciones en un entorno dinámico deben contar con la capacidad de renovarse, de modificar sus habilidades y capacidades, formar redes e innovar para satisfacer a sus clientes y las 
demandas cambiantes del mercado en entornos altamente competitivos (Zapata y Mirabal, 2018).

Las organizaciones, en su mayoría, operan en un ambiente de constantes cambios en cuanto a tecnología, mercado, ciclos más cortos del producto y mucha competencia (Romero, Tejeida y Badillo, 2018). Para Romero y cols. (2018), el desarrollo sustentable es una herramienta competitiva, la cual es revisada y evaluada por la organización dada la presión que ejercen los consumidores, asociaciones civiles, gobierno, entre otros grupos de interés, por lo que es adoptada por estas como una filosofía y estrategia a largo plazo, los beneficios económicos para la empresa, el bienestar social de la comunidad y el cuidado del medio ambiente como un triple beneficio, lo cual ellos definen como Responsabilidad Social Corporativa (RSC), también llamada RSE por Cuatrecasas y González (2017).

Este concepto implica un cambio en la concepción de la organización y su cultura, que expande sus intereses al desarrollo de la localidad (Vera y Rodríguez, 2020). Puentes y Lis-Gutiérrez (2018), señalan que la RSE es la responsabilidad que toman las empresas respecto a los impactos que ocasionan en la sociedad. Autores como Baumgartner y Ebner, citado en Solís y cols., 2017) definen sustentabilidad corporativa como la integración del desarrollo sustentable en la organización con sus tres pilares: económico, social y ambiental. Cuatrecasas y González (2017) toman la definición del Libro Verde de la Comisión Europea en donde se define la RSE como "la integración voluntaria por parte de las empresas las preocupaciones sociales y medioambientales en sus operaciones comerciales y sus relaciones con sus interlocutores" (p.279).

Entre las ventajas de adoptar las prácticas de responsabilidad social están la capacidad de adaptarse a los cambios, mejorar la posición competitiva de la organización, beneficios económicos y sociales, estabilidad y sostenibilidad en el tiempo. Es decir, una empresa sustentable está orientada a la implementación de prácticas organizacionales éticas que responden a los retos planteados por la sociedad y el medio ambiente, basan su visión más allá del crecimiento meramente financiero y se ocupan del crecimiento de la comunidad y de la conservación del medio (Cuevas, Rocha y Soto, 2016).

En su investigación Figueroa y García (2018) indican que, los grupos de interés (stakeholders) alrededor de una organización son un elemento muy importante para tomar en cuenta hablando de prácticas de sustentabilidad corporativa, porque pueden llegar a motivar -o presionar- a los tomadores de decisiones a inclinarse por acciones social y ambientalmente responsables, mediante valoraciones que afecten la imagen de la empresa o impacten sus ventas. León y Afcha (2018), encontraron en su investigación que los grupos de interés relacionados con la cadena de valor (proveedores, clientes, competidores), el medio ambiente y la dirección corporativa, son las que mayor influencia tienen sobre las acciones de RSE.

Romero y cols. (2018) y Puentes y Lis-Gutiérrez (2018), añaden ventajas para las organizaciones que se conducen bajo premisas socialmente responsables, como lo son: ahorro de costos, oportunidad de crecimiento, fidelidad, confianza y mejor relación con los clientes, acceso a financiamientos, uso eficiente de recursos, una mejor imagen corporativa, rentabilidad a largo plazo, guía para la toma de decisiones y posicionamiento de la marca.

Sin embargo, la cultura de la RSC no se ha interiorizado en todas las organizaciones, porque existe la disyuntiva acerca de invertir en sustentabilidad o en rentabilidad (Carro, Sarmiento y Rosano, 2017) y a pesar de los beneficios que pudiera traer a las organizaciones, existen barreras para su adopción como el tiempo que se tarda la incorporación de la cultura en la organización, la limitación de recursos humanos y financieros, la estructura organizativa y la baja percepción e información incorrecta acerca de la RSC (Romero y cols., 2018). Y aunque las prácticas de responsabilidad social no son obligatorias, las organizaciones las asumen como ventaja competitiva para mantenerse en el mercado (Lizcano-Prada y Lombana, 2018).

Por su parte, Chávez e Ibarra (2016) y Velázquez y Vargas-Hernández (2012) aseguran que la competitividad está en función de la sustentabilidad, pues adoptar prácticas de sustentabilidad económica, social y ambiental se torna en una ventaja competitiva a largo plazo, por lo que la consideran indispensable para la supervivencia de una organización.

Finalmente, Cuatrecasas y González (2017) señaIan que la calidad y la RSC están relacionadas en el sentido de que el cliente no valora solamente el cumplimiento de los requisitos del producto sino también las condiciones en que este es elaborado, como resultado de los cambios en el entorno que 
han ocasionado un aumento en el consumo socialmente responsable.

\section{RESULTADOS Y DISCUSIÓN}

Acerca del concepto calidad se puede observar su evolución y adaptación a través del tiempo en la Figura 2. Luego del análisis de los aportes de revisión del concepto calidad, se define de la siguiente manera: calidad es el conjunto de características tangibles e intangibles (Schönsleben, 2019) de un producto o servicio que se adecuan a las exigencias del mercado y que juntas ofrecen al consumidor la satisfacción de sus necesidades explícitas e implícitas (Kotler y Keller, 2012).
En cuanto a los aportes de la Teoría de las Convenciones sobre las percepciones de la calidad, es interesante cómo estas son entendidas de diversas maneras por los consumidores y contribuyen a ampliar las perspectivas de las organizaciones para la satisfacción de los requisitos del cliente. Particularmente, dentro de las convenciones cívicas de la percepción de la calidad, que incluyen aspectos que generen un bien a los demás, pueden incluirse las demandas intangibles del mercado o demandas que tienen que ver con prácticas de responsabilidad económica, social o ambiental; y dentro de las convenciones industriales, que se rigen por el cumplimiento de normas y estándares, pueden incluirse las certificaciones de productos y procesos.

\section{Principales características}

- Detección y solución de problemas a través de la medición y pruebas de los productos para evitar que lleguen con defectos a manos del consumidor

- Se pasa de la inspección en masa a la inspección por muestreos el uso de métodos de control estadístico y gráficos de control.

- Prevención más que detección para el control de calidad.

- Se involucra a todos los departamentos de la organización en la elaboración de políticas de calidad.

- Control Total de Calidad: se procura la calidad tanto en productos como en servicios

- Énfasis en el mercado y necesidades del consumidor. - La calidad se convierte en una estrategia de competitividad.

Calidad Total

(hacia 1990)

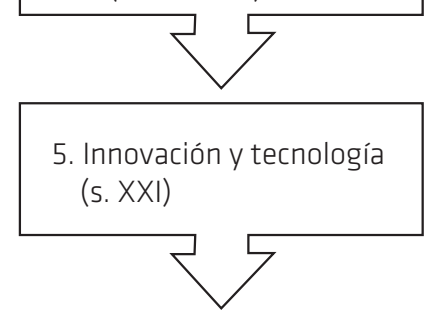

- Constante innovación de productos, procesos y servicios.

- Uso de tecnologías de procesose información para responder rápidamente a los cambiosdel mercado.

Figura 2. Etapas de evolución de la calidad. Fuente: elaboración propia según revisión de literatura. 
En cuanto a los hallazgos en la literatura sobre demanda intangible de mercado, esta es definida como aquella exigencia de aspectos no visibles en el producto final, que están relacionados con la sustentabilidad económica, social y ambiental (Solís y cols., 2017; Terán y cols., 2019).

Con estos fundamentos se puede decir que la calidad ha de configurarse como una demanda intangible de mercado con sustento en los hallazgos de este estudio, y se puede definir como: el conjunto de características tangibles e intangi- bles (Schönsleben, 2019) de un producto o servicio que se adecuan a las exigencias del mercado y que juntas ofrecen al consumidor la satisfacción de sus necesidades explícitas e implícitas (Kotler y Keller, 2012), que tienen que ver con aspectos relacionados a la sustentabilidad social, ambiental y económica (Solís y cols., 2017; Terán y cols., 2019).

En la Tabla 3 se resumen las principales demandas intangibles relacionadas con las tres dimensiones de la sustentabilidad encontradas en este estudio exploratorio.

Tabla 3. Resumen de demandas intangibles de mercado.

\begin{tabular}{|c|c|c|}
\hline \multicolumn{3}{|c|}{ Demandas de responsabilidad social o demandas intangibles de mercado } \\
\hline Dimensión económica & Dimensión Social & Dimensión Ambiental \\
\hline $\begin{array}{l}\text { Bienestar económico de los grupos } \\
\text { de interés, economía de la región, } \\
\text { desarrollo de proveedores, certifi- } \\
\text { caciones (Lizcano-Prada y Lomba- } \\
\text { na, 2018) }\end{array}$ & $\begin{array}{l}\text { Creación de empleos directos e indirec- } \\
\text { tos (Romero et al., 2018) }\end{array}$ & $\begin{array}{l}\text { Preservación y cuidado del medio ambiente, } \\
\text { disminución de contaminación ambiental, uso } \\
\text { de tecnologías limpias (Zarta, 2018) }\end{array}$ \\
\hline $\begin{array}{l}\text { Impacto a la comunidad y desa- } \\
\text { rrollo regional (Aguilera y Puerto, } \\
\text { 2012; Pérez-Batres, 2011) }\end{array}$ & $\begin{array}{l}\text { Apoyo en la formación de empleados, } \\
\text { incorpora en sus decisiones los intere- } \\
\text { ses de la comunidad (Esparza y Reyes, } \\
\text { 2019) }\end{array}$ & $\begin{array}{l}\text { Uso de hormonas y químicos en la agricultu- } \\
\text { ra y ganadería (Hernández y Villaseñor, 2014; } \\
\text { Turgut et al., 2011) }\end{array}$ \\
\hline $\begin{array}{l}\text { Prácticas empresariales de colabo- } \\
\text { ración (Esparza y Reyes, 2019) }\end{array}$ & $\begin{array}{l}\text { Derechos de los trabajadores y de los } \\
\text { pueblos (Hernández y Villaseñor, 2014) }\end{array}$ & $\begin{array}{l}\text { Uso de recursos naturales de forma eficiente, } \\
\text { gestión de suelos y uso de agua (Lizcano-Pra- } \\
\text { da y Lombana, 2018) }\end{array}$ \\
\hline $\begin{array}{l}\text { Servicios públicos e infraestructura } \\
\text { (Romero et al., 2018) }\end{array}$ & $\begin{array}{l}\text { Equidad de género (Dempsey et al., } \\
\text { 2011; Terán et al., 2019) }\end{array}$ & Uso de energías renovables (Fouquet, 2012) \\
\hline $\begin{array}{l}\text { Innovación y tecnología (Gavito et } \\
\text { al., 2017) }\end{array}$ & $\begin{array}{l}\text { Inclusión de personas con discapacidad } \\
\text { (Gómez y Peñaranda, 2019) }\end{array}$ & $\begin{array}{l}\text { Prácticas de producción limpia (Calderón y Fló- } \\
\text { rez, 2015) }\end{array}$ \\
\hline \multirow[t]{4}{*}{$\begin{array}{l}\text { Buenos salarios a la comunidad, } \\
\text { mejora de la calidad de vida de } \\
\text { empleados y comunidad, mayores } \\
\text { niveles de productividad (Zarta, } \\
\text { 2018) }\end{array}$} & $\begin{array}{l}\text { Impacto a la comunidad, condiciones } \\
\text { favorables para los trabajadores (Agui- } \\
\text { lera y Puerto, 2012; Pérez-Batres et al., } \\
\text { 2011) }\end{array}$ & $\begin{array}{l}\text { Cumplir y exceder las normas legales, reuti- } \\
\text { lizar y reciclar materiales, uso de tecnologías } \\
\text { alternativas, empaques reciclables (Esparza y } \\
\text { Reyes, 2019) }\end{array}$ \\
\hline & $\begin{array}{l}\text { Protección a los consumidores (Daft, } \\
\text { 2015) }\end{array}$ & \\
\hline & $\begin{array}{l}\text { Comercio justo, inocuidad de los ali- } \\
\text { mentos, producción responsable (Liz- } \\
\text { cano-Prada y Lombana, 2018) }\end{array}$ & \\
\hline & $\begin{array}{l}\text { Trato digno a los trabajadores y clien- } \\
\text { tes, cohesión comunitaria (Zarta, 2018) }\end{array}$ & \\
\hline
\end{tabular}

Fuente: elaboración propia según revisión de literatura.

Acerca del concepto organización sustentable, este queda definido como aquella organización que ha integrado los principios éticos del desarrollo sustentable -económico, social y ambiental(Solís y cols., 2017; Terán y cols., 2019; Zenteno-Hi- dalgo y Durán, 2016). Es decir, es una organización que se ha adaptado en su estructura, procesos, sistemas y métodos a un nuevo modelo que toma en cuenta la sustentabilidad como estrategia competitiva, acogiéndola dentro de su cultura 
organizativa (Kent, 2020), que le traerá ventajas de mejor imagen corporativa, rentabilidad a largo plazo, fidelidad del cliente, guía para la toma de decisiones, posicionamiento de la marca, estabilidad a largo plazo, permanencia en el mercado, crecimiento empresarial y un desarrollo regional sustentable.

Lo anterior aunado al señalamiento de Cuatrecasas y González (2017) acerca de que la calidad y la RSC están relacionadas desde el momento en que el cliente valora el producto como el proceso desde un enfoque socialmente responsable, lleva a la reflexión acerca de si una organización sustentable es aquella que adopta los principios de sustentabilidad por las presiones de clientes cada vez más exigentes en cuanto a demandas intangibles de mercado (demandas de sustentabilidad económica, social y ambiental).

\section{COMENTARIOS FINALES}

Esta investigación cumple con los objetivos de recopilar, analizar y sistematizar la información publicada sobre el estado del conocimiento de temas de calidad, demanda intangible de mercado y organizaciones sustentables, así como discutir la asociación entre estos constructos teóricos.

De este modo, se evidencia la postura de diversos autores que configuran la calidad como demandas intangibles de sustentabilidad, las cuales son mejor explicadas a la luz de la Teoría de las Convenciones que aporta criterios para valorar la calidad de los productos bajo sus seis convenciones, aportando significativos indicios para que los productores atiendan y se ajusten a las demandas del mercado.

La presente investigación aporta evidencia de la relación entre calidad y organizaciones sustentables que aportan conocimientos para abordar problemáticas de las organizaciones modernas, lo cual sugiere la importancia de esta línea de investigación. Por último, hay que señalar que la calidad como demanda intangible de mercado es un término que se va adecuando y proporciona una nueva vertiente que permite comprender y aportar elementos conceptuales al estudio de las organizaciones en su tránsito hacia la sustentabilidad.

\section{REFERENCIAS}

Aguilera y Puerto. (2012). Crecimiento empresarial basado en la responsabilidad social. Pensamiento \& Gestión, 32, 1-26.

Amezcua, Briseño, Ríos y Ayala. (2018). La disposición a pagar más por productos vinculados a la RSE: evidencia de un análisis conjunto en México. Contaduría y Administración, 63(2), 1-21.

Araya y Rojas. (2020). Consumo responsable e intención de compra en sectores populares: una aproximación multivariante. Ciencias Administrativas, 16, 13-24.

Araya-Pizarro y Araya. (2020). Importancia de la certificación de calidad en la decisión de compra de productos agroalimentarios artesanales. Innovar, 30(77), 53-62.

Boltanski, L. y Thévenot, L. (1991). De la justification. Les économies de la grandeur. Paris: Gallimard.

Calderón y Flórez. (2015). Valoración y análisis de indicadores de sostenibilidad en seis unidades de producción agropecuaria de la cuenca media del Río Chinchiná. Luna Azul, 41, 73-88.

Cantú. (2011). Desarrollo de una cultura de calidad. McGraw-Hill.

Carro, Sarmiento y Rosano. (2017). La cultura organizacional y su influencia en la sustentabilidad. Estudios gerenciales, 33, 352-365.

Chávez e Ibarra. (2016). Liderazgo y cambio cultural en la organización para la sustentabilidad. Telos. Revista de Estudios Interdisciplinarios en Ciencias Sociales, 18(1), 138-158.

Cidell. (2012). Building quality, building green: Conventions Theory and industry transformation. Urbani Izziv, 23(2), 186-194.

Contreras-Valenzuela, Preciado-Rodríguez, Báez-Sañudo, Robles-Parra, Taddei-Bringas y Valderrain. (2018). Certificaciones agrícolas como conducta estratégica del sistema vid de mesa sonorense. Revista Iberoamericana de Tecnología Postcosecha, 19(1), 4-20.

Cuatrecasas y González. (2017). Gestión integral de la calidad: implantación, control y certificación. Profit Editorial. 
Cuevas, Rocha y Soto. (2016). Incentivos, motivaciones y beneficios de la incorporación de la gestión ambiental en las empresas. Universidad o Empresa, 18(30), 121-141.

Daft. (2015). Teoría y diseño organizacional. CENGAGE Learning.

Dempsey, Bramley, Power y Brown. (2011). The social dimension of sustainable development: Defining urban social sustainability. Sustainable Development, 19(5), 289-300.

Esparza y Reyes. (2019). Prácticas de responsabilidad social empresarial desarrolladas por empresas familiares mexicanas y su efecto en el éxito competitivo y la innovación. Tec Empresarial, 13(2), 45-57.

Figueroa y García. (2018). Un modelo para la toma de decisiones sustentables en las organizaciones. Investigación administrativa, 48(122), $1-17$.

Fischer y Espejo. (2011). Mercadotecnia. McGraw-Hill.

Fouquet. (2012). The demand for environmental quality in driving transitions to low-polluting energy sources. Energy Policy, 50, 138-149.

Gavito, Van der Wal, Aldasoro, Ayala-Orozco, BuIlén, Cach-Pérez, Casas-Fernández, Fuentes, Conzález-Esquivel, Jaramillo-López, Martínez, Masera-Cerruti, Pascual, Pérez-Salicurp, Robles, Ruiz-Mercado y Villanueva. (2017). Ecología, tecnología e innovación para la sustentabilidad: retos y perspectivas en México. Revista Mexicana de biodiversidad, 88, 150160.

Giménez, Jiménez y Martínez. (2014). La gestión de calidad: importancia de la cultura organizativa para el desarrollo de variables intangibles. Revista Europea de Dirección y Economía de la Empresa, 23, 115-126.

Gómez y Peñaranda. (2019). El estado del arte de la responsabilidad social empresarial e inclusión laboral de las personas con discapacidad. Espacios, 40(22), 19.

Gutiérrez Pulido. (2014). Calidad y productividad. McGraw-Hill.

Hernández y Villaseñor. (2014). La calidad en el sistema agroalimentario globalizado. Revista Mexicana de Sociología, 76(4), 557- 582.
International Organization for Standarization. (s.f.). Recovered from: https://www.ipax.gob. mx Kent. (2020). Gestión y evaluación de la sustentabilidad organizacional. Ciencias Administrativas, 15, 1-12.

Kotler y Keller. (2012). Dirección de Mercadotecnia. Pearson Educación.

León y Afcha. (2018). Incidence of stakeholders in the corporate social responsibility of micro, small and medium-sized enterprises of Santa Marta. Cuadernos de administración, 34(62), 3-19.

Liyanagel y Warahena. (2019). The Recognition of Intangible Quality Aspects: a topic for the Next Generation. Eighth Intl. Conf. on Advances in Social Science, Management and Human Behaviour - SMHB.

Lizcano-Prada y Lombana. (2018). Enfoques de la responsabilidad social empresarial en los agronegocios. Estudios gerenciales, 34(148), 347-356.

Mankiw, N. G. (2017). Principios de Economía. Cengage Learning Editores.

Palma-Perez, Arteaga-Flores, Ponce-Andrade, Loor-Chávez, Calderón y Mejía. (2018). El marketing sensorial y su influencia en el comportamiento de compra de los consumidores de la ciudad de Manta, Ecuador. Dominio de las ciencias, 4(4), 145-158.

Pérez-Batres, Miller y Pisani. (2011). Institutionalizing Sustainability: An Empirical Study of Corporate Registration and Commitment to the United Nations Clobal Compact Guidelines. Journal of cleaner production, 19(8), 843851.

Platas y Cervantes. (2017). Gestión integral de la calidad. Un enfoque por competencias. Grupo Editorial Patria.

Puentes y Lis-Gutiérrez. (2018). Medición de la responsabilidad social empresarial: una revisión de la literatura (2010-2017). Suma de negocios, 9(20), 145-152.

Robles y Garza. (2011). Nuevas condicionantes en las organizaciones de los sistemas alimentarios: el caso del sistema vid de mesa de Sonora. En L. Huesca, M. Camberos y C. Calderón, Bienestar y desarrollo en el siglo XXI, 189-203. CIAD/Plaza y Valdés Editores. 
Romero, Tejeida y Badillo. (2018). El sistema de responsabilidad social corporativa como fundamento de la innovación en PYME turísticas. Nueva época, 8(17), 327-354.

Rossi, Devós, De Oliveira y Baker. (2020). Development and validation of a scale for identification of quality attributes of agri-food products in short chains. Geoforum (111), 165-175.

Ruiz, Castelló, Climent, Escalona, Hernández, Loscertales y Frutos. (2013). La calidad del vino a la luz de la teoría de las convenciones: aplicación a las denominaciones de origen aragonesas. Estudios Geográficos, 74(274), 231-254.

Sanabria, Romero y Flórez. (2014). El concepto de calidad en las organizaciones: una aproximación desde la complejidad. Universidad o Empresa, 16(27), 165-213.

Schönsleben. (2019). Tangible services and intangible products in industrial product service systems. 11th CIRP Conference on Industrial Product-Service Systems, IPS2 2019. Zhuhai \& Hong Kong, China.

Solís, Robles, Preciado y Hurtado. (2017). El papel del mercado en la construcción de organizaciones sustentables. Estudios sociales, 27(49), 273-294.

Tarziján y Paredes. (2012). Organización industrial para la estrategia empresarial. Pearson.

Terán, Robles, Preciado y López. (2019). Equidad gerencial como demanda intangible de mercado. Entre ciencia e ingeniería, 13(26), 85-93.
Turgut, Ornek y Cutright. (2011). Determination of pesticide residues in Turkey's table grapes: the effect of integrated pest management, organic farming, and conventional farming. Environmental Monitoring and Assessment, 173, 315-323.

Velázquez y Vargas-Hernández. (2012). La sustentabilidad como modelo de desarrollo responsable y competitivo. Ingeniería de recursos naturales y del ambiente, 11, 97-107.

Vera y Rodríguez. (2020). Aspectos conceptuales sobre la relación entre el desarrollo territorial y responsabilidad social. En L. P. Almeida. A dialética das desigualdades sociais (págs. 253-266). Editora UCDB.

Zapata y Mirabal. (2018). Capacidades dinámicas de la organización: revisión de literatura y un modelo propuesto. Investigación administrativa, 47(121).

Zarta. (2018). La sustentabilidad o sostenibilidad: un concepto poderoso para la humanidad. Tabula Rasa, 28, 409-423.

Zenteno-Hidalgo y Durán. (2016). Factores y prácticas de alto desempeño que influyen en el clima laboral: análisis de un caso. Innovar, 26(59), 119-135.

Zinoubi. (2020). Determinants of consumer purchase intention and behavior toward green products: the moderating role of price sensitivity. Archives of Business Research, 8(1), 261273. 